\title{
pneumonia
}

Review

\section{Reflections on pneumonia in the tropics}

\author{
Michael P Alpers \\ Faculty of Health Sciences, Curtin University, Perth, Australia
}

Corresponding author: Prof Michael P Alpers, Room 108, Health Research Campus, Shenton Park, Curtin University, GPO Box U1987, Perth, Western Australia 6845, Australia. Phone +61 (0)8 9266 4733. Email: m.alpers@curtin.edu.au

Author contribution: M.P.A. participated in the design, implementation of all activities and analysis of data and in the writing of the manuscript. This author contributed entirely to the writing of this paper.

Received Dec 2, 2013; Accepted Jan 22, 2014; Published Mar 16, 2014

Citation: Alpers MP. Reflections on pneumonia in the tropics. pneumonia 2014;4:1-7.

\section{Abstract}

This review of pneumonia in the tropics is based on experience with respiratory infections in Papua New Guinea since the 1970s. It discusses ideas, principles, historical aspects of pneumonia research and the need to work with people in the community. In order to understand pneumonia in a tropical setting and evaluate new interventions it is essential to study the ecosystem of the causative infections, within the host and the community and between interacting microorganisms. Vaccines are much-needed preventive tools, and for pneumonia in a highly endemic setting the prevention of severe and fatal disease takes priority over the prevention of infection. In this setting mild infection plays an important role in preventing severe disease. For achieving long-term sustainable outcomes, sometimes 'less is more'. A multipronged approach is required to control and prevent pneumonia, and in devising new ways of doing so. This includes appropriate and accessible clinical care, a clean, smoke-free environment, good nutrition and a range of vaccines. Also required are persistent advocacy from the global scientific community and strong engagement with and by the communities that bear the burden of disease. Better health care must be pursued in conjunction with raising literacy rates and reducing poverty.
\end{abstract}

Keywords: pneumonia, nasopharyngeal carriage, pneumococcal polysaccharide vaccine, ecosystem of infection, community engagement

The following observations and reflections derive from my experiences working with many creative and committed scientists across a range of disciplines investigating pneumonia and other tropical infectious diseases at the Papua New Guinea Institute of Medical Research, of which I was the Director from 1977 to 2000.

One of the first things we learned in our studies of infectious disease in Papua New Guinea was that to understand an infection we had to investigate the ecosystem in which it occurred. Later we learned that the results of an intervention depended on the specifics of the ecosystem in which it was applied.

Following the pioneering work of Robert Douglas [1] and lan Riley [2] in the 1970s we undertook studies on pneumonia [3] within a comprehensive set of research programs [4]. We looked for the invading organisms in the blood and lungs $[5,6]$. We also investigated the ecosystem inhabited by these organisms, which at the time was considered an 
unusual approach. We studied the nasopharynx $[7,8]$, the first acquisition of pathogenic organisms after birth [9] and the complex relationships of acquisition, carriage and invasiveness [10]. Bacterial pathogens were acquired remarkably early in life and high levels of bacterial carriage persisted in the nasopharynx throughout childhood. We also studied the ecosystem of the household and the response to an intervention against the principal source of respiratory pathogens in the household, namely, chronic bronchitis in adults [11].

Streptococcus pneumoniae (pneumococcus) and Haemophilus influenzae, together with a number of viruses such as respiratory syncytial virus, were the principal respiratory pathogens found. The pneumococcus and $H$. influenzae were also important causes of meningitis [12-14]. These organisms were of established significance world-wide and the subject of much past and ongoing research. The first trial of a 7-valent pneumococcal conjugate vaccine in the United States demonstrated a powerful specific effect in children [15], which was widely acclaimed, and a dramatic effect of herd immunity in protecting unvaccinated adults from pneumococcal disease [16]. These encouraging results were demonstrated in the population for which the vaccine was designed. In other populations, however, the outcome was somewhat different [17]. Such a difference in another cultural, ethnic and ecological setting was entirely to be expected, at least by those who worked in tropical communities of the Third World.

The conjugate pneumococcal vaccine has not been rolled out yet in Papua New Guinea though a country-wide program will be established with the 13 -valent vaccine in 2014. We know that the coverage of pathogenic serotypes by the vaccine will be less than in the First World. We can expect significant replacement infection and disease, probably more than occurred in Alaskan Native Americans [17]. We do not expect to get herd immunity, since this can only occur where the community source of infection is the group that has been immunised, in this case young children. Where, in contrast, the route of transmission goes the other way, and children are principally infected by adults living in their household, adults will not be protected by immunising infants. Indeed, any gaps in the nasopharyngeal niche of children created by vaccination will readily be filled by locally circulating pathogenic types not in the vaccine. That is why, apart from its benefit in improving the health of adults, we were keen to implement the oral vaccine - or immune modulator - that reduced bronchial bacterial load [11], and the health services of Papua New Guinea were strongly supportive of this initiative. Unfortunately the manufacture of the vaccine ceased. Further studies of a new formulation have since been undertaken and we still hope that it may become available again [18].

In trying to modify the ecosystem to the benefit of the host it may be more sustainable in the long run to use a minor perturbation rather than a major disruption that evokes a powerful reactive adjustment to maintain ecological stability. In this respect the use of a vaccine that does not affect nasopharyngeal carriage and provides immunity against a broad range of pathogenic serotypes may be ultimately advantageous. On first principles there is an advantage in filling the niche of infection in the nasopharynx with organisms to which the host is immune, to the extent of being able to mount a rapid and effective response against invasive disease. These bacteria will reduce nasopharyngeal infection by other competing organisms and if they invade will be dealt with by host immunity before they cause severe and fatal disease. The polysaccharide pneumococcal vaccine has been shown to provide such protection [19]. This benefit can be achieved for children aged 9 months and over - the belief that the polysaccharide vaccine is non-immunogenic and ineffective in children under the age of 2 years is still trotted out as dogma, even though in tropical populations of the Third World it is manifestly untrue $[20,21]$. For children under the age of 9 months some form of protein-based vaccine is required, whether a set of protein-polysaccharide conjugates covering specific serotypes, a combination of species-specific pneumococcal proteins or a whole cell vaccine. We have to expect that counterstrategies by the pneumococcus, either replacement with other serotypes or proliferation of variant strains, will diminish the initial vaccine efficacy. Some bacterial vaccine interventions 
have been singularly successful and we must keep trying with established and new technologies, such as reverse vaccinology, but we also need to temper our optimism with humility. In our human arrogance we tend to think of bacteria as primitive organisms and forget that they have been evolving and adapting in a very competitive world for aeons longer than we have.

For $H$. influenzae, type b stands out as the most virulent serotype and $H$. influenzae type b (Hib) vaccine has had dramatic effects in reducing Hib disease wherever it has been used, including Papua New Guinea. However, Hib is not the only serotype of $H$. influenzae causing disease [22] and type a disease may be becoming more common in Papua New Guinea. Whether this proves to be a serious problem will be determined by ongoing surveillance at the Papua New Guinea Institute of Medical Research. Nontypeable $H$. influenzae are also important organisms whose role in disease requires further investigation [23]. We do not understand the complexities of infection and bacterial association among different species and strains, pathogenic and non-pathogenic, within the nasopharynx but we do know that if a niche is empty it will not remain so but be filled by other organisms.

Another important principle that we learned in Papua New Guinea is the importance of mild infection [24]. This was the principle of variolation, which predated the concept of attenuated infection through vaccination, which is a cognate principle and generally a safer practice. However, in the absence of a vaccine, mild infection plays an important role in preventing fatal disease. It cannot be guaranteed but can be promoted by strategies to reduce the infecting dose and strengthen the immune system, through various environmental and nutritional improvements. Chronic mild infection is particularly important in malaria, where it helps to maintain adult immunity in malaria-endemic areas. The combination blood-stage vaccine against malaria that we devised and tested was intended not to have any effect on infection but to prevent severe disease and death from malaria. This principle was designed for the vaccination of children in an endemic area and proof of the principle was demonstrated in a Phase $2 b$ vaccine trial where density of infection was used as a surrogate for disease severity $[25,26]$. The same principle applied to our work on pneumonia. The trial of the 23-valent pneumococcal polysaccharide vaccine showed no effect on mild pneumonia but a $58 \%$ efficacy against death from pneumonia in children aged 6 months to 5 years and, because of the importance of pneumonia as a cause of death in this population, a $30 \%$ reduction in all-cause mortality [19]. An effect on severe morbidity was also demonstrated [27] but only when the burden was high during a pneumonia epidemic. Even in children aged 6 months to 2 years there were significant effects of the vaccine.

Because of the high burden of severe disease and mortality from pneumonia in young children we wanted to start administering the vaccine in Papua New Guinea as soon as possible, but the country could not afford it. However, its cost of manufacture was not high and, given a global market, we expected that the price could have been brought down to an affordable level. Thus our first move after conducting this trial in the Papua New Guinean highlands was to try and have it replicated elsewhere. This was done with the full support of the World Health Organization (WHO), which had supported the trial itself. Somewhat ironically, because of a high expectation of its efficacy, WHO had imposed methodological constraints on the study, insisting on sequential analysis so that at the first evidence of efficacy the trial could be stopped and the vaccine given to the control group. This gave a clear outcome but significantly compromised our ability to undertake more in-depth analysis of the results. With WHO support a replicate trial was designed in The Gambia. However, our colleagues engaged in the development of a conjugate pneumococcal vaccine persuaded the funders of this trial that it was not worth pursuing because the more powerful conjugate pneumococcal vaccine was 'just around the corner'. This was in 1989: 24 years later Papua New Guinean children still have not received the benefit of pneumococcal vaccination. During that period how many lives could have been saved from the use of the 23-valent pneumococcal polysaccharide vaccine in Papua New Guinea and in other countries of the Third World with 
similar ecological characteristics?

The development of vaccines against complex infections has much in common with the deployment of antibiotics. The original hype that the use of antibiotics would soon eliminate infectious disease, an opinion expressed even by leaders in biological science, was based on ignorance of the adaptability of microorganisms. Even scientists seem to forget that bacteria have a long experience of dealing with antibiotics in their environment. In the 1970s we conducted a study in a remote part of Papua New Guinea looking for bacterial resistance to antibiotics that had never been used in the participating communities. As a result we isolated several gut organisms that were resistant to these antibiotics. Though this was what some of our team had predicted, the finding was at the time considered controversial, to the unfortunate extent that our more conservative colleagues would not agree to its publication.

In some infections, such as with a specific virus that infects only humans, eradication is theoretically and, indeed, in the case of smallpox, practically possible. This is not so for complex infections and diseases such as malaria or pneumonia. If the infection has low endemicity or is epidemic it may be possible to eliminate it in that location. Where eradication is not possible or has not been achieved, elimination is dangerous, since it sets up an open niche with absent or waning host immunity, or creates other kinds of vulnerability. This is true across a range of infections from polio to malaria. Elimination is worth it if constant and rigorous surveillance, which does not come cheaply, can be provided to prevent the reintroduction of the infection and deal immediately with any resurgence of disease. Where overall elimination is not possible, as with respiratory infections, and good control is the ideal, it may be a sound investment to include in the long-term strategy a component that leads to minimal perturbation of the relevant ecosystem. In this respect we may anticipate a continuing future role for polysaccharide pneumococcal vaccination in the control of pneumonia. This is especially so if the vaccine is offered at a price that low-income countries can sustainably afford - which is, unfortunately, unlikely to happen with conjugate vaccines.
The multiplicity of pathogenic serotypes in the ecosystem in tropical communities is a significant risk factor and warrants pneumococcal capsular vaccines of high valency; the 23-valent vaccine could possibly be expanded for this purpose and the dose of each component reduced. However, this need may change when the long-awaited species-specific protein vaccines have been licensed and their impacts evaluated: dare we hope that this is just around the corner'?

In recent studies in Papua New Guinea the 23-valent pneumococcal polysaccharide vaccine has been used to provide boosting (at age 9 months) of responses initiated at birth or in early infancy by conjugate vaccine [28]. The results have shown that neonatal immunisation is safe and immunogenic [29] and immunologically safe, with good T-cell responses induced [30]. Immunisation at birth has two main advantages: it enables high coverage through making use of the best opportunity for immunisation, at the time of birth in a health facility; and it provides the earliest possible protection to infants. This is particularly relevant in places like Papua New Guinea where over half of the deaths from pneumonia in children aged less than 5 years occur in the first six months of life [31]. To achieve early protection we also tried immunising mothers with pneumococcal vaccine in pregnancy [32]. Though this gave encouraging results, it has not yet been followed up with efficacy studies to determine the extent to which maternal immunisation actually protects young infants from disease. A major concern is how to prevent the rapid colonisation of these infants with multiple respiratory pathogens, which is a risk factor for early severe disease - and otitis media - and may also have deleterious immunological effects. A probiotic approach with benign competing organisms would be worth pursuing against both the pneumococcus and haemophilus, and recent clinical [33] and experimental [34] studies with the pneumococcus have shown encouraging results. We would also like to try enhancing mucosal immunity with an infant formulation of the oral haemophilus 'vaccine' that we successfully tested in adults.

Boosting of early conjugate vaccine responses by the 23-valent pneumococcal polysaccharide vaccine at 9 
months was clearly shown [29]. The positive primary response at this age to serotypes not included in the conjugate vaccine provided further evidence to dispel the myth of the non-immunogenicity of polysaccharide vaccine under the age of 2 years. Similar results were found in Fiji [21], where the functionality of these antibodies was also established [35]. The Fiji study raised the question of hyporesponsiveness following boosting by polysaccharide vaccine [36]. Whether these responses are what one would expect from a well-regulated immune system or lower than expected (hyporesponses) is a matter for debate. Furthermore, it is not clear what the state of 'hyporesponsiveness' means, since it is not immunological tolerance in the strict sense. Further detailed investigation of the immune responses, including cellular effects, after pneumococcal antigenic challenge is needed. These aspects are included in the protocol of new studies underway in Papua New Guinea. However, though a high load of polysaccharide antigen does have immunological risks [37] there is no evidence that the dosage of the pneumococcal polysaccharide vaccine used for boosting has been associated with any deleterious clinical effects.

One of the principles of research conducted by the Papua New Guinea Institute of Medical Research has been to ensure access to the best available clinical care for all participants. A further principle is that when an innovative intervention is identified it should be evaluated as soon as possible and, if found to be efficacious, implemented which, as we have seen, is usually easier said than done. The collective power of the scientists who meet every two years at the International Symposium on Pneumococci and Pneumococcal Diseases (ISPPD) and exchange information on all aspects of pneumococcal science is most impressive. However, there was no mechanism for harnessing this power for the immediate benefit of those who were suffering from pneumonia, the disease most commonly caused by the pneumococcus. We addressed this deficiency at ISPPD5 held in Alice Springs in 2006. A draft Global Action Plan against Pneumonia (GAPP) was presented to and discussed by the Symposium [38]. It became the forerunner of several other Global Action Plans promoted by international agencies. This promotion is active and has expanded to include diarrhoea [39]. However, though good plans make a start, actually getting action is a slow process and can easily lose momentum. The GAPP has remained on the agenda of subsequent ISPPDs and will be discussed again in 2014 in Hyderabad, India. This issue must not be lost sight of and requires continual pushing since pneumonia is ever the forgotten disease or the one that is simply taken for granted. The issue is also surprisingly controversial: when I presented the idea of the GAPP in Alice Springs, the first reaction of a senior colleague was "Why would you want to do that?". Other colleagues were in general supportive of the idea but wanted to push only for vaccines as the means of closing the gap between mortality rates from pneumonia in poor and underprivileged communities and those in affluent ones. All available methods need to be implemented ready access to good clinical management and appropriate antibiotics when disease occurs and prevention through a healthy environment, good nutrition and vaccines.

One of the most difficult things to achieve in closing this gap is community engagement. Policy-makers and people generally find it difficult to understand that pneumonia is the major killer of children globally and particularly in the hot tropics, where most of these deaths occur. Tropical communities also ignore pneumonia but for the opposite reason: children dying of pneumonia is so common that it is considered a fact of life that one can do nothing about and simply has to accept. To persuade communities and the individuals who live in them that this is not acceptable is a major first step in getting the community engaged in its own health and well-being. Offering accessible clinical care, at a health service facility or in the community, helps, but change will be slow until the community actively pursues ways of improving their own health. Only when the community begins to demand better access to lifesaving health care and persists with its demands will deaths from pneumonia significantly decline. Preventive measures such as vaccines and improved household environment and nutrition are part of this care. Enhanced community engagement requires a long-standing dialogue and reciprocal understanding between health services and the community. Strong and committed individuals are 
needed on both sides. Parents can be taught to recognise when they have a seriously sick child but this recognition has no value if clinical help is not readily accessible. If the staff of a well-equipped clinic have no engagement with the community, there will continue to be frequent deaths from pneumonia in that community. For the future of the community, education and economic opportunity are also vitally important. Nor can the local effects of global environmental change be ignored [40]. To deal with these interfaces we need a new breed of health worker, with similar counterparts in other sectors, and active participation from the community. We have the knowledge and tools already to make a difference to the burden of pneumonia. As well as seeking new knowledge and better tools we need to implement more effectively those that we have. Strengthening community engagement, which extends far beyond pneumonia, marks the final frontier of pneumonia research in the tropics.

\section{Acknowledgements}

I acknowledge the research colleagues in respiratory infection with whom I have worked closely over many years. Most - but by no means all - of them may be found in the references below. I thank colleagues teaching in the Medical School, determining health policy or delivering health care in Papua New Guinea, for their friendship and support. I acknowledge and remember with pleasure the many interactions and animated discussions with leaders and people in the communities who participated in our studies. I have been privileged to learn from them all. For this paper I acknowledge the encouragement and help of both in-house and external reviewers.

\section{Funding: None}

\section{Competing interests: None}

Copyright: This is an open access article distributed under the terms of the Creative Commons Attribution License 3.0, which permits unrestricted use, distribution, and reproduction in any medium, provided the original author and source are credited.

\section{References}

1. Douglas RM. Pneumonia in Papua New Guinea, from the past to the future. PNG Med J 2010;53(3-4):99-105.

2. Riley ID. Pneumonia research in Papua New Guinea: 19671986. PNG Med J 2010;53(3-4):106-18.

3. Alpers MP. The pneumonia research program of the Papua New Guinea Institute of Medical Research: its beginnings and the development of an integrated approach. In: Leahy C, Vilakiva G, Diave D, eds. Action against Pneumonia: A Celebration of 40 Years of Pneumonia Research in PNG and Finding the Best Way Forward. Goroka: Papua New Guinea Institute of Medical Research, 2010:21-26.

4. Alpers MP. Past and present research activities of the Papua New Guinea Institute of Medical Research. PNG Med J 1999;42(1-2):32-51.

5. Shann F, Gratten M, Germer S, Linnemann V, Hazlett D, Payne R. Aetiology of pneumonia in children in Goroka Hospital, Papua New Guinea. Lancet 1984;2(8402):537-41.

6. Barker J, Gratten M, Riley I, Lehmann D, Montgomery $\mathrm{J}$, Kajoi $\mathrm{M}$ et al. Pneumonia in children in the Eastern Highlands of Papua New Guinea: a bacteriologic study of patients selected by standard clinical criteria. J Infect Dis 1989;159(2):348-52.

7. Montgomery JM, Lehmann D, Smith T, Michael A, Joseph $B$, Lupiwa $T$ et al. Bacterial colonization of the upper respiratory tract and its association with acute lower respiratory tract infections in highland children of Papua New Guinea. Rev Infect Dis 1990;12(Suppl 8):S1006-16.

8. Lehmann D, Gratten M, Montgomery J. Susceptibility of pneumococcal carriage isolates to penicillin provides a conservative estimate of susceptibility of invasive pneumococci. Pediatr Infect Dis J 1997;16(3):297-305.

9. Gratten M, Gratten H, Poli A, Carrad E, Raymer M, Koki G. Colonisation of Haemophilus influenzae and Streptococcus pneumoniae in the upper respiratory tract of neonates in Papua New Guinea: primary acquisition, duration of carriage, and relationship to carriage in mothers. Biol Neonate 1986;50(2):114-20.

10. Smith T, Lehmann D, Montgomery J, Gratten M, Riley ID, Alpers MP. Acquisition and invasiveness of different serotypes of Streptococcus pneumoniae in young children. Epidemiol Infect 1993;111(1):27-40.

11. Lehmann D, Coakley KJ, Coakley CA, Spooner V, Montgomery $J M$, Michael A et al. Reduction in the incidence of acute bronchitis by an oral Haemophilus influenzae vaccine in patients with chronic bronchitis in the highlands of Papua New Guinea. Am Rev Respir Dis 1991;144(2):324-30.

12. Gratten M, Barker J, Shann F, Gerega G, Montgomery J, Kajoi M, Lupiwa T. The aetiology of purulent meningitis in highland children: a bacteriological study. PNG Med J 1985;28(4):233-40.

13. Gratten M, Montgomery J. The bacteriology of acute pneumonia and meningitis in children in Papua New Guinea: assumptions, facts and technical strategies. PNG Med J 1991;34(3):185-98.

14. Lehmann D, Yeka W, Rongap T, Javati A, Saleu G, Clegg A et al. Aetiology and clinical signs of bacterial meningitis in children admitted to Goroka Base Hospital, Papua New Guinea, 1989-1992. Ann Trop Paediatr 1999;19(1):21-32.

15. Black $S$, Shinefield H, Fireman B, Lewis E, Ray P, Hansen JR et al. Efficacy, safety and immunogenicity of heptavalent pneumococcal conjugate vaccine in children. Northern California Kaiser Permanente Vaccine Study Center Group. Pediatr Infect Dis J 2000;19(3):187-95.

16. O'Brien KL, Dagan R. The potential indirect effect of conjugate pneumococcal vaccines. Vaccine 2003;21(1718):1815-25.

17. Singleton RJ, Hennessy TW, Bulkow LR, Hammitt LL, Zulz T, Hurlburt DA et al. Invasive pneumococcal disease caused by nonvaccine serotypes among Alaska native children with high levels of 7-valent pneumococcal conjugate vaccine 
coverage. JAMA 2007;297(16):1784-92.

18. Clancy R. Collaborative studies in mucosal immunology in Goroka. PNG Med J 2010;53(3-4):122-25.

19. Riley ID, Lehmann D, Alpers MP, Marshall TF, Gratten H, Smith D. Pneumococcal vaccine prevents death from acute lower-respiratory-tract infections in Papua New Guinean children. Lancet 1986;2(8512):877-81.

20. Pomat WS, Lehmann D, Sanders RC, Lewis DJ, Wilson J, Rogers $S$ et al. Immunoglobulin $G$ antibody responses to polyvalent pneumococcal vaccine in children in the highlands of Papua New Guinea. Infect Immun 1994; 62(5):1848-53.

21. Balloch A, Licciardi PV, Russell FM, Mulholland EK, Tang MLK. Infants aged 12 months can mount adequate serotypespecific IgG responses to pneumococcal polysaccharide vaccine. J Allergy Clin Immunol 2010;126(2):395-97.

22. Gratten M, Barker J, Shann F, Gerega G, Montgomery J, Kajoi M, Lupiwa T. Non-type b Haemophilus influenzae meningitis. Lancet 1985;1(8441):1343-44.

23. Cripps AW. Nontypeable Haemophilus influenzae and childhood pneumonia. PNG Med J 2010;53(3-4):147-50.

24. Alpers MP. The importance of mild infection in controlling severe disease in areas of high endemicity: mild infection as a vaccine strategy. In: Leahy C, Vilakiva G, Diave D, eds. Action against Pneumonia: A Celebration of 40 Years of Pneumonia Research in PNG and Finding the Best Way Forward. Goroka: Papua New Guinea Institute of Medical Research, 2010:62-63.

25. Genton B, Betuela I, Felger I, Al-Yaman F, Anders RF, Saul A et al. A recombinant blood-stage malaria vaccine reduces Plasmodium falciparum density and exerts selective pressure on parasite populations in a phase $1-2 b$ trial in Papua New Guinea. J Infect Dis 2002;185(6):820-27.

26. Flück C, Smith T, Beck HP, Irion A, Betuela I, Alpers MP et al. Strain-specific humoral response to a polymorphic malaria vaccine. Infect Immun 2004;72(11):6300-05.

27. Lehmann D, Marshall TF, Riley ID, Alpers MP. Effect of pneumococcal vaccine on morbidity from acute lower respiratory tract infections in Papua New Guinean children. Ann Trop Paediatr 1991;11(3):247-57.

28. Phuanukoonnon S, Reeder JC, Pomat WS, van den Biggelaar AHJ, Holt PG, Saleu $G$ et al. A neonatal pneumococcal conjugate vaccine trial in Papua New Guinea: study population, methods and operational challenges. PNG Med J 2010;53(3-4):191-206.

29. Pomat WS, van den Biggelaar AHJ, Phuanukoonnon $S$, Francis J, Jacoby P, Siba PM et al. Safety and immunogenicity of neonatal pneumococcal conjugate vaccination in Papua New Guinean children: a randomised controlled trial. PloS One 2013;8(2):e56698.
30. van den Biggelaar AHJ, Richmond PC, Pomat WS, Phuanukoonnon S, Nadal-Sims MA, Devitt CJ et al. Neonatal pneumococcal conjugate vaccine immunization primes $\mathrm{T}$ cells for preferential Th2 cytokine expression: a randomized controlled trial in Papua New Guinea. Vaccine 2009;27(9):1340-47.

31. Kakazo M, Lehmann D, Coakley K, Gratten H, Saleu G, Taime $J$ et al. Mortality rates and the utilization of health services during terminal illness in the Asaro Valley, Eastern Highlands Province, Papua New Guinea. PNG Med J 1999;42(1-2):13-26.

32. Lehmann D, Pomat WS, Combs B, Dyke T, Alpers MP. Maternal immunization with pneumococcal polysaccharide vaccine in the highlands of Papua New Guinea. Vaccine 2002;20(13-14):1837-45.

33. John M, Dunne EM, Licciardi PV, Satzke C, Wijburg O, Robins-Browne RM, O'Leary S. Otitis media among highrisk populations: can probiotics inhibit Streptococcus pneumoniae colonisation and the risk of disease? Eur J Clin Microbiol Infect Dis 2013;32(9):1101-10.

34. Wong SS, Toh ZQ, Dunne EM, Mulholland EK, Tang MLK, Robins-Browne RM et al. Inhibition of Streptococcus pneumoniae adherence to human epithelial cells in vitro by the probiotic Lactobacillus rhamnosus GG. BMC Res Notes 2013;6:135.

35. Licciardi PV, Balloch A, Russell FM, Burton RL, Lin J, Nahm $\mathrm{MH}$ et al. Pneumococcal polysaccharide vaccine at 12 months of age produces functional immune responses. J Allergy Clin Immunol 2012;129(3):794-800.

36. Russell FM, Carapetis JR, Balloch A, Licciardi PV, Jenney AW, Tikoduadua $L$ et al. Hyporesponsiveness to re-challenge dose following pneumococcal polysaccharide vaccine at 12 months of age: a randomized controlled trial. Vaccine 2010;28(19):3341-49.

37. O'Brien KL, Hochman M, Goldblatt D. Combined schedules of pneumococcal conjugate and polysaccharide vaccines: is hyporesponsiveness an issue? Lancet Infect Dis 2007;7(9):597-606.

38. Cripps AW, Leach AJ, Lehmann D, Benger N. Meeting report: Fifth International Symposium on Pneumococci and Pneumococcal Diseases, Alice Springs, Central Australia, 2-6 April 2006. Vaccine 2007;25(13):2361-65.

39. World Health Organization/The United Nations Children's Fund (UNICEF). Ending Preventable Child Deaths from Pneumonia and Diarrhoea by 2025. The Integrated Global Action Plan for Pneumonia and Diarrhoea (GAPPD). Geneva: World Health Organization, 2013.

40. Alpers MP. Some general factors to be considered when implementing a program to control pneumonia. PNG Med J 2010;53(3-4):94-98. 